\title{
Aumento de reborde alveolar por medio de distracción osteogénica para la colocación de implantes
}

Gálvez y Gálvez E. Aumento de reborde alveolar por medio distracción osteogénica para la colocación de implantes. Rev Estomatol Herediana. 2008; 18(1):44-49.

\section{RESUMEN}

La reconstrucción de rebordes alveolares atróficos por medio de la técnica de distracción osteogénica alveolar (DOA) ofrece un resultado previsible con bajas tasas de morbilidad y una ganancia notable de tejido óseo y tejidos blandos, en comparación con las técnicas tradicionalmente utilizadas, así también éste método nos permite optimizar las posiciones de los implantes tridimensionalmente en el tejido óseo logrado. Este trabajo describe el tratamiento de un paciente de sexo masculino de 20 años de edad quien presentaba atrofia severa del reborde alveolar en la región anterior del maxilar por medio de DOA, se utilizo un dispositivo yuxtaoseo. Comenzando la activación del dispositivo a los 8 días posteriores a la instalación, con un patrón de activación de $0.6 \mathrm{~mm}$. diarios hasta alcanzar la altura ósea deseada, se retiró el distractor, teniendo un periodo de consolidación de 20 semanas y posteriormente la coloración de implantes oseointegrados, pudo comprobarse clínica y radiográficamente el incremento en altura y volumen óseo necesario para la rehabilitación y satisfacer las necesidades funcionales y estéticas.

\section{Palabras claves: OSTEOGÉNESIS POR DISTRACCIÓN / IMPLANTES DENTALES.}

Increase in size of alveolar ridges by means of distraction osteogenesis for implante placement

ABSTRACT

The reconstruction of atrophic alveolar ridges by means of the technique of alveolar distraction osteogenesis (ADO) offers a foregone result with small morbidity rates and a remarkable gain of bony and soft tissues, in comparison with the traditionally used techniques. A 20 year old male patient who presented a severe atrophy of alveolar ridge in the maxillary anterior region was assisted by means of ADO, using a juxtaosseous device, beginning the activation of the device, 8 days after the installation, with a pattern of activation of $0.6 \mathrm{~mm}$. per day until reaching the wanted bony height. The distractor was removed and the implants were placed 20 weeks later. It was found clinically and radiographically, the gain of the height and necessary bony volume for the rehabilitation with implants.

Key words: DISTRACTION OSTEOGENESIS / DENTAL IMPLANTS.

\section{Eduardo Gálvez y Gálvez}

${ }^{1}$ Cirujano - Dentista

\section{Correspondencia}

Eduardo Galvez y Galvez.

ulio C. Tello 1020 - Lima 14

Telefono: 470-4559/998-464402

e-mail: galvezygalvez@ hotmail.com

Recibido : 5 de mayo del 2008

Aceptado : 30 de junio del 2008

\section{Introducción}

Las causas mas comunes de la pérdida ósea del reborde alveolar son la enfermedad periodontal, trauma dentoalveolar o deformidades congénitas. Para la rehabilitación por medio de implantes oseointegrados el receptor debe ofrecer un lecho con un volumen y una altura ósea que permita la adecuada estabilidad primaria y posición favorable en función a las piezas por restituir. La utilización de injertos de hueso autógeno, así como el uso de membranas y materiales aloplásticos son los métodos tradicionalmente utilizados en la reconstrucción alveolar, siendo ampliamente descrita la alta tasa de morbilidad y de reabsorción ósea en la región, además de no ofrecer resultados previsibles, y de necesitar un tiempo de espera entre la cirugía para aumento de reborde y la colocación de los implantes de aproximadamente seis meses.

La distracción osteogénica alveolar (DOA) es una alternativa, consiste en una técnica de alargamiento del callo óseo obtenido por osteotomía previa. Los segmentos óseos obtenidos son separados de manera controlada y continua, creando una tensión en el callo óseo que estimula la osteogenesis y correspondientemente el crecimiento de los tejidos blandos, la distracción oseogénica ha sido considerada como la única estrategia capaz de corregir los defectos óseos y de los tejidos blandos circundantes simultáneamente, confiriéndoles las dimensiones (aumento de la altura y ancho del reborde alveolar) y propiedades biomecánicas deseables.
Ofreciendo un resultado previsible, con bajas tasas de morbilidad e infección, así como un periodo de espera significativamente menor para la colocación de los implantes (10 semanas), en comparación con los métodos tradicionalmente utilizados.

Históricamente, fue Codivilla, en 1905, el primero en describir la técnica de distracción osteogénica para el alargamiento de los miembros inferiores. Luego Ilizarov (1989) fue quien realizo la mayor parte de las investigaciones clínicas y biológicas relacionadas con esta técnica. Mccormick, en Kurgan (Rusia), despues de la segunda guerra mundial, desarrolló un sistema de fijación externa en forma de aros para que fueran utilizados en el tratamiento de fracturas óseas.

Block, aplicó estos principios a 
nivel experimental, siendo el primero en publicar estudios sobre el uso de la DOA en animales en 1996. Ese mismo año, Chin y Toth reportan el uso clínico de la DOA como tratamiento de deficiencias de reborde alveolar en el maxilar superior.

La DOA es una técnica que nos permite aumentar la altura del reborde alveolar promoviendo la neoformación ósea, así como un aumento significativo de los tejidos blandos circundantes.

Actualmente la distracción osteogénica se aplica en el tratamiento de diversas deformaciones craneofaciales, tales como correcciones de los defectos en el tercio medio de la cara, en el alargamiento anterior de la maxila, aumento del largo y ancho del hueso alveolar, corrección de los defectos complejos del esqueleto facial mediante la distracción bio o multifocal.

Los diversos tipos de dispositivos de DOA comercialmente disponibles pueden clasificarse básicamente en dos grupos: los yuxtaoseos y los intraoseos. Los dispositivos intraóseos son colocados a traves del segmento de transporte en la dirección del vector de distracción, en cambio, los dispositivos yuxtaoseos son fijados por medio de tornillos monocorticales al disco de transporte y a la cortical externa de la mandíbula o maxilar, ofreciendo una mayor estabilidad del disco de transporte en la etapa de distracción y de consolidación ósea.

\section{Etapas de la distracción osteogénica}

1. Osteotomía e instalación del distractor. En esta primera etapa se hace la completa transección del hueso (incluyendo el periostio, cortical y medular ). A continuación se procede con la instalación de un distractor adecuado, vecino al sitio de la fractura, con el objetivo de eliminar cualquier tipo de movimiento entre los segmentos óseos obtenidos en la transección. El grado de estabilidad local dependerá del tipo de distractor y del modo de instalación del mismo. Pequeñas inestabilidades en la fijación del conjunto pueden perjudicar la angiogenésis y causar la formación de fibrocartílago. La fijación estable y la manutención de la función muscular local proporcionan la formación de callo óseo en corto periodo de tiempo.

2. Periodo de latencia. Es el periodo comprendido entre la cirugía, para la realización de la transección e instalación del distractor, y el movimiento de inicio de la distracción propiamente dicha. En este periodo, ocurre en el foco de transección la formación de un coagulo que es posteriormente sustituido por el tejido de granulación.

Parece no haber consenso en lo que se refiere al periodo de latencia, habiendo una variación de 0 a 14 días. No obstante, el tiempo de latencia ideal parece estar relacionado con una serie de factores, tales como la edad y el tipo de transección.

En situaciones clinicas se ha relatado que un periodo de latencia de siete días parece ser mas eficiente; no obstante en pacientes jóvenes se recomienda un periodo de latencia de dos a cinco días y de siete a catorce días en pacientes mayores, en las áreas con poco hueso, lechos de pobre perfusión vascular o cuando hubo gran trauma quirúrgico.

3. Periodo de activación-aplicación de fuerzas de distracción. En este período se hace la aplicación de las fuerzas de distracción en los segmentos generados por la corticotomía, causando el alarga- miento del callo óseo, fase en la que se debe considerar el índice y el ritmo de distracción.

El índice de distracción es el numero de milímetros que se tensiona el segmento óseo por día. El índice de 1 milímetro parece ideal para la formación de tejido óseo.

El ritmo de la distracción esta determinado por el número de eventos de distracción por día. El índice de 1 mm puede ser aplicado en un único momento o ser dividido en dos o en cuatro eventos de distracción al día, con $0,5 \mathrm{~mm}$ y $0,25 \mathrm{~mm}$ de distracción, respectivamente. Generalmente los mejores resultados en el alargamiento de los tejidos se observa con mayor frecuencia en varios episodios de distracción.

4. Periodo de consolidación. El periodo de consolidación se sitúa entre el final de la distracción y la retirada del distractor. Despues del alargamiento, el dispositivo debe permanecer en el lugar para garantizar la inducción de la osificación y la consecuente consolidación de la fractura.

En este periodo, la estabilidad del distractor es factor relevante para la formación del regenerado óseo, ya que la movilidad excesiva puede causar la formación de fibrosis. Aunque varios tipos de dispositivos estén disponibles para el alargamiento del tejido óseo, hay que destacar en cualquier situación el fijador debe ser rígido y compacto para permitir la formación ósea y el bienestar del paciente.

El examen radiográfico de un foco de distracción osteogénico demuestra que despues del termino de la distracción de los segmentos proximal y distal del callo se tornan calcificados y fusionados. El callo óseo resultante es gradualmente consolidado y finalmente remodelado 
Los modelos experimentales de la formación de nuevo tejido óseo primario ocurre dos semanas despues del inicio de la distracción, de forma simultanea se inicia el proceso de mineralización. Después de la segunda semana, las trabéculas finas presentan características de remodelado, tornándose mas gruesas. Tres semanas despues del inicio de la distracción, la mineralización se presenta en etapa mas avanzada .

En la cuarta semana, la formación ósea progresa, con aumento de hueso lamelar orientado según la dirección de la tracción; no obstante, la zona central permanece esencialmente con las mismas características durante todo el proceso de distracción. Cuatro semanas después del termino de la distracción se observa competa continuidad entre los dos segmentos óseos y, hasta 20 semanas, el hueso regenerado esta formado y consolidado.

\section{Biología de la distracción osteogénica}

Ilizarov perfeccionó el método de distracción y demostró que el efecto de una fuerza de tensión contínua y controlada, aplicada a los segmentos de la transección, induce la neovascularización local, la proliferación, la diferenciación y la posición de células a lo largo del eje de la fuerza aplicada. La tensión orientada estimula el crecimiento, no solamente del tejido óseo, sino también de las partes blandas. La confirmación de estos descubrimientos llevo a Ilizarov a concluir que la aplicación de las fuerzas de tracción generán tensiones que estimulan la histogenésis.

La regeneración de los tejidos bajo la influencia de la distracción recuerda el crecimiento embrionario y la consolidación de la fractura.
En el foco de la distracción, la formación ósea es precedida por la formación del coagulo, infiltrado inflamatorio y tejido de granulación.

En este tejido, el aumento de la celularidadad se da a causa de las células mesenquimaticas fenotípicamente semejantes a los fibroblastos maduros que depositan fibras colágenas que se organizan en la matriz extracelular.

Bajo condiciones ideales la fijación, en el foco de la distracción el tejido neoformado evoluciona para un proceso de osificación intramenbranosa, sin la formación de tejido cartilaginoso.

Durante la distracción osteogénica han sido identificados solamente regiones focales, en estas áreas no se observó la presencia del colágeno tipo II en la matriz ósea en distracción, indicando que la fase de fibrocartílago no se presenta. Muchos estudiosos atribuyen la presencia del cartílago a disturbios en la vascularización focal, con disminución de de la tensión de oxígeno y alteración del proceso de formación ósea de mesenquimal para endocondral.

Estos disturbios pueden ser intrínsecos del tejido o atribuidos a la fijación externa insuficiente, permitiendo micromovimientos y consecuentemente daños a la vascularización local y baja tensión de oxigeno.

Un hecho interesante de destacarse es que el suministro vascular de la cabeza y del cuello es superior al suministro de las extremidades, debido a la formación de los arcos aórticos en el periodo embrionario. Ha sido sugerido que el suministro vascular abundante de estas topografías tenga importante participación en el proceso de osificación directa.

Si el tejido del cayo y los capilares se desarrollan en el mismo rit- mo, las células osteoprogenitoras se diferencian en un ámbito vascularizado y formarán el hueso. Si hubiera proporcionalmente pocos vasos o movimiento de los segmentos de la fractura, ocurriría la formación de cartílago y posterior sustitución de esta por el tejido óseo (osificación endocondral ). No obstante, si el movimiento es excesivo y el suministro vascular escaso, habría una gran posibilidad del establecimiento de una fibrosis.

Durante el proceso de distracción ocurren importantes ganancias de tejido decurrentes de las fuerzas de tensión aplicadas sobre los segmentos óseos. Las partes blandas, incluyendo la epidermis, dermis, los vasos sanguíneos, los tendones, los músculos y los nervios acompañan el crecimiento óseo, resultando en completa interacción fisiológica entre los tejidos blandos y las estructuras óseas.

\section{Reporte del caso}

Paciente masculino de 20 años de edad, aparentemente sano, y sin alteraciones sistémicas, atendido en el Clínica Dental Docente de la Facultad de Estomatología de UPCH, el cual presento deficiencia del reborde alveolar en región anterior del maxilar, siendo causada por traumatismo dentoalveolar con perdida de las piezas 11, 21.

Adicionalmente el paciente presentaba una maloclución tipo III (13 años de edad). Fue atendido por el Servicio de Ortodoncia, el cual corrigió el plano oclusal y la relación intermaxilar buscando la nivelación del plano oclusal y armonizando oclusión intermaxilar.

Luego fue realizada la colocación de un dispositivo de DOA bajo anestesia local (solución de Lidocaína al $2 \%$ y epinefrina 1:100.000 IU). El distractor utilizado fue el tipo 
yuxtaoseo (Conexao Implant System ${ }^{\circledR}$ - SP-Brasil).

Fué realizó una incisión horizontal 5mm. por debajo de la cresta del reborde alveolar con la elevación de un colgajo de espesor total exponiendo la cortical vestibular. Posteriormente se realizaron dos osteotomías verticales y divergentes entre si por medio de una sierra oscilante con una pieza recta y motor eléctrico bajo irrigación constante con solución fisiológica, abarcando la cortical hasta llegar al hueso medular y posteriormente completadas por medio de un cincel recto. Una tercera osteotomía horizontal fue realizada apical a los dos cortes verticales obteniéndose el segmento de transporte, siendo finalmente colocado el distractor y la fijación del mismo por medio de tornillos monocorticales de $8 \mathrm{~mm}$ de largo por 1,5 de diámetro y posteriormente la activación para determinar el desplazamiento libre del segmento de transporte así como la ausencia de trabas mecánicas (Fig. 1). Se retornó el distractor a su posición inicial y se realizó el reposicionamiento pasivo del colgajo y la síntesis por medio de sutura reabsorbible 4.0 mediante puntos simples. Se indicó como medicación pos-operatorio Ketorolaco de 10mg cada 8 horas por 3 días y Clindamicina $300 \mathrm{mg}$, cada 12 horas por 5 días, además de enjuagatorios con gluconato de clorhexidina al $0,12 \% 2$ veces al día por dos semanas.

Después de 8 días de un periodo de latencia se inicio la activación del distractor previo a un control radiográfico. Se aplicó un patrón de activación a razón de dos veces al día (cada vuelta 0.33 ), llegando a
$0.66 \mathrm{~mm}$ diarios por un periodo de 22 días. Luego de un periodo de consolidación de 12 semanas se procedió al retro del distractor.

Después de 20 semanas de la instalación del distractor, se realiza la colocación de los implantes (Renova, Lifecore). En la planificación de la ubicación de los implantes se realizo un encerado de diagnóstico, basado en la Proporción Divina, para determinar el espacio requerido de los dos centrales faltantes, luego con esas medidas se fabrico una prótesis provisional que nos sirvió a la vez de férula topográfica (Fig. 2). Con la determinación del ancho de los incisivos centrales se observo la presencia de un mayor espacio del requerido (2,6 $\mathrm{mm}$ ) haciéndose necesario posteriormente la Mesialización del segundo cuadrante para obtener una correc-
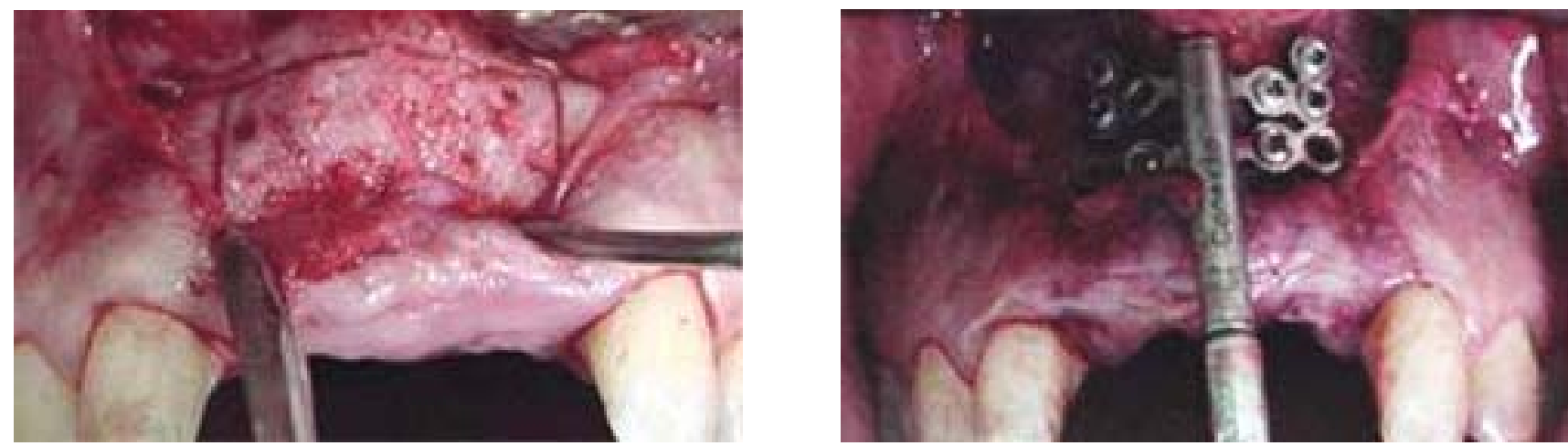

Fig. 1. Izquierda: Realización de las osteotomías verticales y horizontales para la obtención del segmento de transporte. Derecha: Posicionamiento y fijación del distractor.
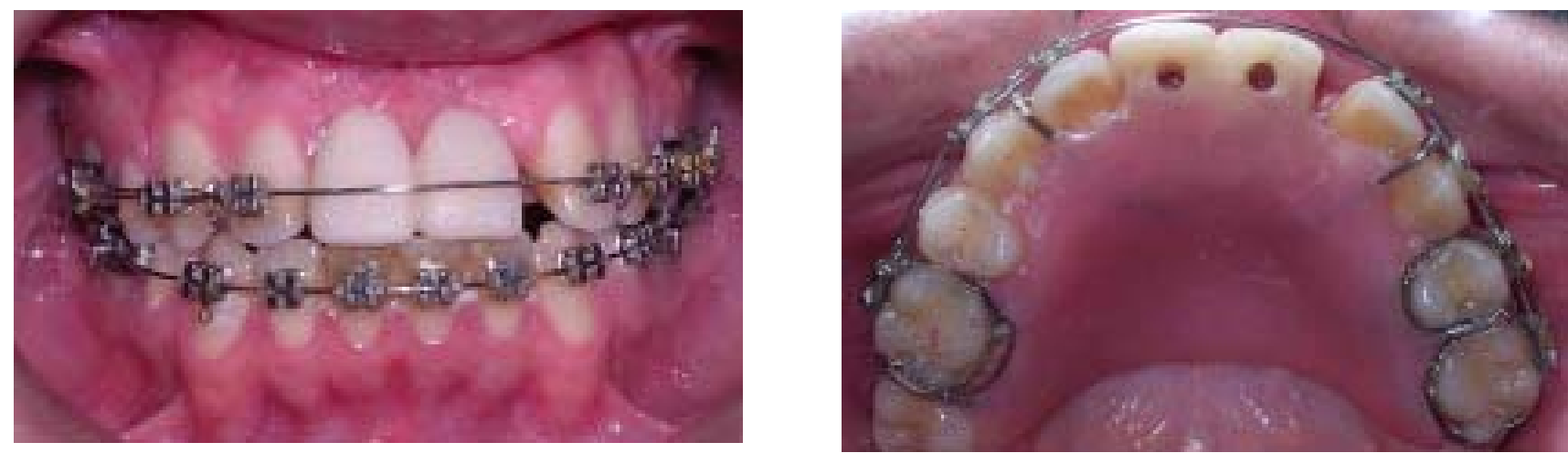

Fig. 2. Confección de prótesis acrílica, remplazando los incisivos centrales con el tamaño adecuado según la referencia del incisivo lateral. Obsérvese la mayor presencia del espacio requerido, haciéndose necesaria la mesialización de las piezas (2,6 mm) del segundo cuadrante, mediante el tratamiento ortodóntico. 
ta alineación a cargo del servicio de ortodoncia

\section{Resultados}

Clínica y radiográficamente se observó un aumento en altura y espesura del reborde alveolar (Fig. 3 y 4), obteniéndose un aumento óseo de $9 \mathrm{~mm}$ logrando una óptima estabilidad primaria de los implantes. No hubo evidencia clínica de alteración sensorial o reabsorción a nivel del reborde alveolar. Tanto en los posoperatorios de la primera y segunda fase quirúrgica no se observaron señales de infección.

\section{Discusión}

La utilización de la DOA en la reconstrucción de rebordes alveolares atróficos demostró ser un método que ofrece un incremento en la al- tura del reborde alveolar por medio de aumento óseo y de tejidos blandos, adecuando el reborde para una posterior rehabilitación por medio de implantes, siendo un método previsible y con bajas tasas de reabsorción ósea, en comparación con el uso de injertos óseos o materiales aloplásticos. Adicionalmente ofrece un menor tiempo de espera entre la etapa inicial y la colocación de implantes (20 semanas) en comparación con los 6 meses que usualmente deben aguardarse en el caso de utilizarse injertos de hueso autógeno. Podemos concluir que la DOA es una alternativa de tratamiento innovadora y confiable que se traduce en un evaluación costobeneficio favorable tanto para el paciente como para el cirujano.

\section{Referencias bibliográficas}

1. von Arx T, Hardt N, Wallkamm B. The TIME technique: a new method for localized alveolar ridge augmentation prior to placement of dental implants. Int $\mathrm{J}$ Oral Maxillofac Implants. 1996; 11(3):387-94

2. Caplanis N, Sigurdsson TJ, Rohrer MD, Wikesjö UM. Effect of allogeneic, freeze-dried, demineralized bone matrix on guided bone regeneration in supraalveolar peri-implant defects in dogs. Int $\mathrm{J}$ Oral Maxillofac Implants. 1997; 12(5):634-42.

3. Rachmiel A, Srouji S, Peled M. Alveolar ridge augmentation by distraction osteogenesis. Int J Oral Maxillofac Surg. 2001; 30(6):5107.

4. Ilizarov GA. The tension-stress

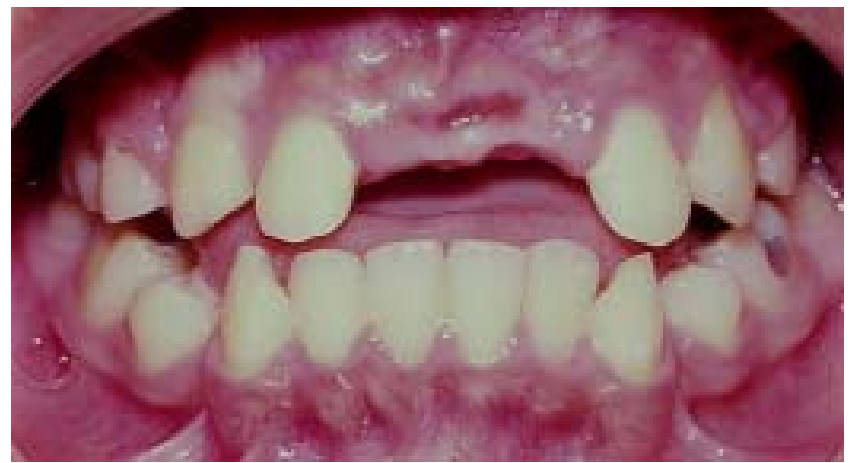

Fig. 3. Imágenes pre y post-operatorias.
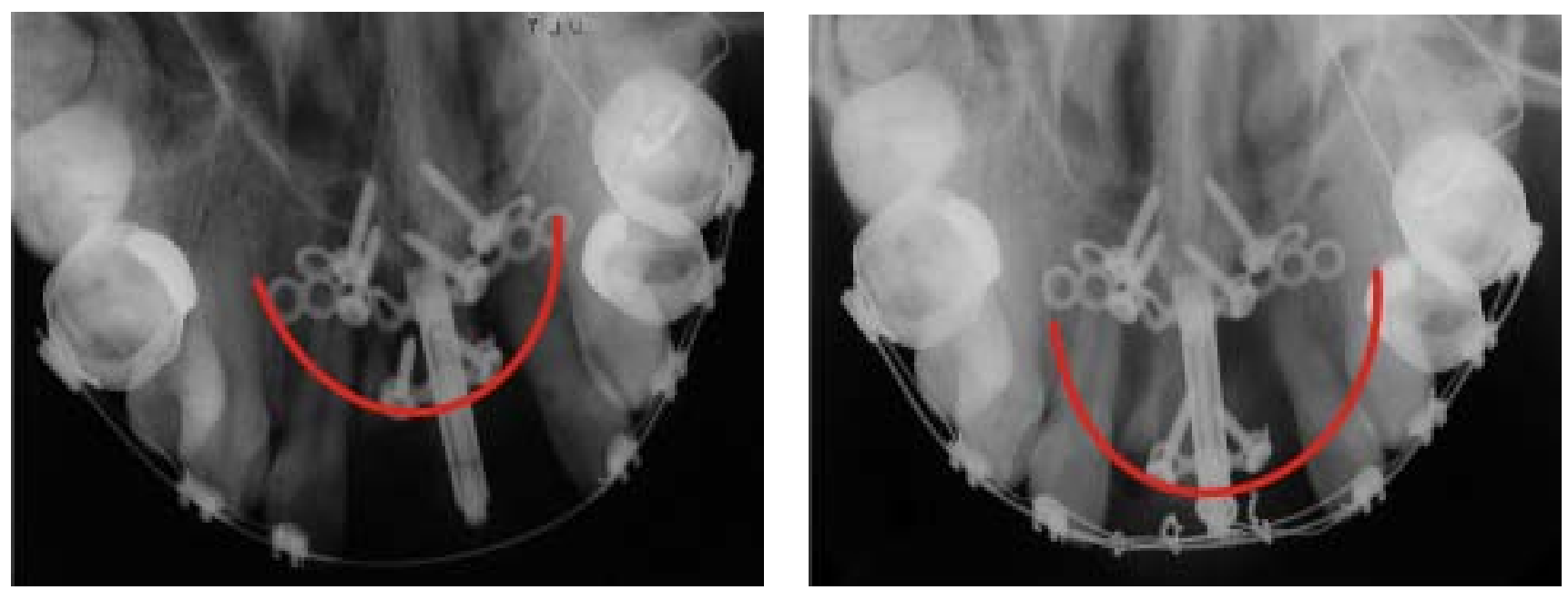

Fig. 4. Imágenes radiográficas pre y post-operatorias donde se aprecia una diferencia notable con respecto a la altura ósea inicial y la obtenida por medio de la DOA. 
effect on the genesis and growth of tissues. Part I. The influence of stability of fixation and soft-tissue preservation. Clin Orthop Relat Res. 1989; (238):249-81.

5. Ilizarov GA. The tension-stress effect on the genesis and growth of tissues: Part II. The influence of the rate and frequency of distraction. Clin Orthop Relat Res. 1989; (239):263-85.

6. Block MS, Almerico B, Crawford C, Gardiner D, Chang A. Bone response to functioning implants in dog mandibular alveolar ridges augmented with distraction osteogenesis. Int J Oral Maxillofac Implants. 1998; 13(3):342-51.

7. Block MS, Chang A, Crawford C. Mandibular alveolar ridge augmentation in the dog using distraction osteogenesis. J Oral Maxillofac Surg. 1996 Mar; 54(3):309-14.

8. Chin M, Toth BA. Distraction osteogenesis in maxillofacial surgery using internal devices: review of five cases. J Oral Maxillofac Surg. 1996; 54(1):4553;

9. Uckan S, Haydar SG, Dolanmaz D. Alveolar distraction: analysis of 10 cases. Oral Surg Oral Med Oral Pathol Oral Radiol Endod. 2002; 94(5):561-5.

10. Gaggl A, Schultes G, Kärcher H. Distraction implants: a new operative technique for alveolar ridge augmentation. J Craniomaxillofac Surg. 1999; 27(4):214-21.

11. Gaggl A, Schultes G Kärcher H. Distraction implants--a new possibility for augmentative treatment of the edentulous atrophic mandible: case report. $\mathrm{Br}$ J Oral Maxillofac Surg. 1999; 37(6):481-5.

12. Garcia AG, Martin MS, Vila PG, Maceiras JL. Minor complications arising in alveolar distraction osteogenesis. J Oral Maxillofac Surg. 2002; 60(5):496-501.

13. Oda T, Sawaki Y, Ueda M. Alveolar ridge augmentation by distraction osteogenesis using titanium implants: an experimental study. Int J Oral Maxillofac Surg. 1999; 28(2):151-6.

14. Hidding J, Lazar F, Zöller JE. [Initial outcome of vertical distraction osteogenesis of the atrophic alveolar ridge] [article in german]. Mund Kiefer Gesichtschir. 1999; 3 Suppl 1:S7983.

15. Klesper B, Lazar F, Siessegger M, Hidding J, Zöller JE. Vertical distraction osteogenesis of fibula transplants for mandibular reconstruction--a preliminary study. J Craniomaxillofac Surg. 2002; 30(5):280-5. 